\title{
Unprovoked pulmonary embolism in older adults: incidence and prognosis
}

Mor Aharoni ${ }^{1,2}$, Nir Horesh ${ }^{1,2}$, Ori Rogowski ${ }^{1,3}$, Anjelika Kremer ${ }^{4}$, Haim Mayan ${ }^{1,5}$, Dan Justo ${ }^{1,4}$

\begin{abstract}
${ }^{1}$ Sackler School of Medicine, Tel-Aviv University, Israel
${ }^{2}$ Surgery Department, Sheba Medical Center, Tel-Hashomer, Israel

${ }^{3}$ Internal Medicine C, Sourasky Medical Center, Tel-Aviv, Israel

${ }^{4}$ Geriatrics Division, Sheba Medical Center, Tel-Hashomer, Israel

${ }^{5}$ Internal Medicine E, Sheba Medical Center, Tel-Hashomer, Israel
\end{abstract}

Submitted: 12 October 2017; Accepted: 30 April 2018

Online publication: 26 February 2021

Arch Med Sci 2021; 17 (2): 337-342

DOI: https://doi.org/10.5114/aoms/90673

Copyright (c) 2021 Termedia \& Banach

\section{Abstract}

Introduction: Unprovoked pulmonary embolism (UPE) is not rare and it is associated with an unfavorable prognosis in adults. However, the incidence and the prognosis of UPE in older adults have never been studied.

Material and methods: This was a historical prospective study. We reviewed all the medical charts of all older adults (aged 70 years or more) with UPE, provoked pulmonary embolism (PPE), and malignancy-associated PE (MAPE), admitted to a tertiary medical center between 2010 and 2012. The all-cause 3 -year mortality rates and cumulative survival following admission were compared between the groups.

Results: The final cohort included 249 patients with PE: 161 (64.7\%) were women; the mean age was $79.8 \pm 5.7$ years. Overall, $36(14.5 \%)$ patients had UPE, 81 (32.5\%) patients had MAPE, and 132 (53.0\%) patients had PPE. Overall, 39 (15.7\%) patients died within 30 days, 76 (30.5\%) patients died within 6 months, 101 (40.6\%) patients died within 1 year, and 136 (54.6\%) patients died within 3 years of admission. Relative to PPE and MAPE patients, the cumulative survival was significantly higher in UPE patients at each time point within 1 year of admission $(p<0.05$ and $p<0.001$, respectively). However, 3 years after admission, the cumulative survival was comparable between PPE patients and UPE patients, and was significantly lower in MAPE patients $(p<0.001)$

Conclusions: UPE is not rare in older adults with PE, and it is associated with a favorable prognosis within 1 year of admission in this population.

Key words: incidence, mortality, older adults, survival, unprovoked pulmonary embolism.

\section{Introduction}

In most patients with pulmonary embolism (PE), the cause or the trigger for thrombosis is apparent and might be an inherited thrombophilia, a recent hip fracture, a recent major operation, hormonal replacement therapy, central lines, a pregnancy, etc. These patients have what is commonly called provoked PE (PPE). Patients with PE caused by an acquired thrombophilia associated with an active malignancy have what is commonly called malignancy-associated PE (MAPE). Idiopathic PE, also called unprovoked pulmonary embolism (UPE), is defined as PE without an obvious cause or a trigger. The diagnosis of UPE in patients with PE is of

\author{
Corresponding author: \\ Dan Justo MD \\ Geriatrics Division \\ Sheba Medical Center \\ Tel-Hashomer 5265601 \\ Israel \\ Phone: 972-52-6668550 \\ Fax: 972-9-7408575 \\ E-mail: dan.justo@sheba. \\ health.gov.il
}


clinical importance since it often calls for an occult malignancy workup, an inherited thrombophilia workup, and longer anticoagulation treatment duration compared with that of PPE patients [1].

The incidence of unprovoked venous thromboembolism (VTE) in adults is $25-40 \%$ [2-4], and the incidence of UPE in adults with PE can be as high as $50 \%$ [1]. However, the incidence of UPE in older adults with PE has never been studied, to the best of our knowledge. Moreover, UPE in adults is associated with a higher mortality rate compared with PPE, and a lower mortality rate compared with MAPE [5-7]. However, mortality rates and cumulative survival associated with UPE, and compared with those of PPE and MAPE, have never been studied in older adults. In view of the worldwide increase in the population of older adults, it is most important to study the incidence of UPE in this population and its associated prognosis.

The aim of the study was to study the incidence of UPE in older adults with PE, and to study the 3 -year mortality rates and cumulative survival in this population.

\section{Material and methods}

\section{Study design}

This was a historical prospective study conducted in Sheba Medical Center - the largest tertiary medical center in Israel, with the approval of the local ethics committee.

\section{Study population}

The medical records of all older adults (aged 70 years or more) with PE who were admitted to Sheba Medical Center between 2010 and 2012 were reviewed. PE was diagnosed in all patients by means of a computed tomography scan with angiography (CTA) of the chest or a lung ventilation-perfusion scan. Patients were divided into three groups according to the causes of PE or their absence: UPE, PPE, and MAPE.

UPE patients were those without obvious causes or triggers for PE. PPE patients were those with obvious causes or triggers for PE including: immobility in the last month following an acute disease or minor surgery or a long-haul flight; immobility in the last 3 months following a debilitating fracture or major surgery or a debilitating stroke; inherited thrombophilia (e.g., history of VTE, family history of VTE, factor V Leiden mutation, Protein $S$ deficiency, etc.); acquired thrombophilia (e.g., essential thrombocytosis, polycythemia vera, nephrotic syndrome, vasculitis, etc.); and use of pro-thrombotic agents (e.g., hormonal replacement therapy, androgens, tamoxifen) [8]. Obese patients, chronically immobile patients, and chronic obstructive lung disease patients, were not included in the PPE group - unless they had other obvious causes or triggers for PE. MAPE patients were those with an active malignancy either treated with chemotherapy and/or metastatic. Patients with an active malignancy diagnosed within 1 year following PE diagnosis were also included in the MAPE group. PPE patients who also had an active malignancy were included in the MAPE group as well.

The preliminary cohort included 275 older adults with PE. We excluded patients in whom PE was highly suspected, but a chest CTA or lung ventilation-perfusion scan was not performed $(n=11$; 4.0\%). We also excluded patients in whom it was impossible to determine whether they had PPE or MAPE $(n=9 ; 3.3 \%)$ and patients with chronic PE $(n=6 ; 2.2 \%)$.

\section{Study variables}

The study variables included age, gender, chronic co-morbidities, mechanical ventilation, discontinuing anticoagulation during hospitalization, all-cause mortality rates and cumulative survival within 30 days, 6 months, 1 year, and 3 years of admission.

\section{Statistical analysis}

The continuous variables were expressed as the mean \pm standard deviation, median, and inter-quartile range (IQR). The one-way Kolmogorov-Smirnov test was used to assess the distribution of continuous variables. Student's t-test was used to compare between the means of continuous variables with parametric distributions. The Mann-Whitney test was used to compare between the means of continuous variables with non-parametric distributions. Fisher's exact test was used to compare between the prevalence/incidence of categorical variables. Kaplan-Meier curves were used to compare cumulative survival between UPE, PPE, and MAPE patients. A stepwise logistic regression analysis was used to study which variables were independently associated with 1-year mortality. Two-tailed $p<0.05$ was considered statistically significant. The statistical analyses were carried out using the $24^{\text {th }}$ version of the SPSS statistical software (IBM, Chicago, IL, USA).

\section{Results}

\section{Descriptive analysis of the whole cohort}

The final cohort included 249 PE patients: most patients ( $n=161,64.7 \%$ ) were women, and the mean age was $79.8 \pm 5.7$ years (median: 80 years; IQR: 75-84 years). The three most common chronic co-morbidities were hypertension, diabe- 
tes mellitus, and ischemic heart disease. Except for $1(0.4 \%)$ patient who was initially treated by thrombolysis, all patients were initially treated with enoxaparin. Except for 10 (4.0\%) patients in whom oral anticoagulation was stopped due to bleeding, $1(0.4 \%)$ patient in whom anticoagulation was stopped due to a terminal cancer, and $1(0.4 \%)$ patient in whom anticoagulation was not started due to sub-segmental PE without a lower limb deep vein thrombosis (DVT), all of the patients were treated with anticoagulation agents upon their discharge. Overall, 18 (7.2\%) patients were mechanically ventilated (Table I).

\section{Incidence of unprovoked PE}

Overall, 36 (14.5\%) patients had UPE, 81 (32.5\%) patients had MAPE, and 132 (53.0\%) patients had PPE. Of note, only 5 (13.9\%) UPE patients underwent a genetic thrombophilia workup - which came back negative for all of them, and none of the UPE patients underwent an occult malignancy workup. Among the 81 MAPE patients, the four most common malignancies were: lung cancer $(n=17 ; 20.9 \%)$, breast cancer $(n=11$; $13.6 \%)$, metastatic disease of an unknown primary $(n=9 ; 11.1 \%)$, and pancreatic cancer $(n=7$; 8.6\%). In 1 (1.2\%) patient an active malignancy was diagnosed within 8 months following PE diagnosis. Except for age, which was significantly higher in UPE patients, and atrial fibrillation, which was significantly more prevalent in PPE patients, there were no statistically significant differences between the three groups in terms of the mean age and the prevalence of other chronic co-morbidities (Table I).

\section{Mortality rates and cumulative survival}

Overall, 39 (15.7\%) patients died within 30 days, 76 (30.5\%) patients died within 6 months, 101 (40.6\%) patients died within 1 year, and 136 (54.6\%) patients died within 3 years of admission. At each time point mortality rates were highest among MAPE patients and lowest among UPE patients. Moreover, none of the UPE patients died within 6 months of admission (Figure 1).

Table I. Clinical characteristics of the whole cohort, MAPE patients, PPE patients, and UPE patients

\begin{tabular}{|c|c|c|c|c|c|}
\hline Variable & $\begin{array}{l}\text { Whole cohort } \\
\qquad(n=249)\end{array}$ & $\begin{array}{l}\text { MAPE } \\
(n=81)\end{array}$ & $\begin{array}{c}\text { PPE } \\
(n=132)\end{array}$ & $\begin{array}{c}\text { UPE } \\
(n=36)\end{array}$ & $P$-value \\
\hline \multicolumn{6}{|c|}{ Demographics and chronic co-morbidities: } \\
\hline $\begin{array}{l}\text { Age, mean } \pm \text { standard } \\
\text { deviation [years] }\end{array}$ & $79.8 \pm 5.7$ & $78.3 \pm 5.1$ & $80.1 \pm 5.7$ & $82.6 \pm 6.1$ & 0.001 \\
\hline Female, $n(\%)$ & $161(64.7)$ & $50(61.7)$ & $84(63.6)$ & $27(75.0)$ & 0.359 \\
\hline Hypertension, $n$ (\%) & $183(73.5)$ & $53(65.4)$ & $101(76.5)$ & $29(80.6)$ & 0.120 \\
\hline $\begin{array}{l}\text { Ischemic heart disease, } \\
n(\%)\end{array}$ & $64(25.7)$ & $20(24.7)$ & $32(24.2)$ & $12(33.3)$ & 0.525 \\
\hline Atrial fibrillation, $n(\%)$ & $37(14.9)$ & $4(4.9)$ & $30(22.7)$ & $3(8.3)$ & 0.001 \\
\hline Dementia, $n(\%)$ & $31(12.4)$ & $7(8.6)$ & $19(14.4)$ & $5(13.9)$ & 0.449 \\
\hline $\begin{array}{l}\text { Congestive heart failure, } \\
n(\%)\end{array}$ & $18(7.2)$ & $6(7.4)$ & $10(7.6)$ & $2(5.6)$ & 0.915 \\
\hline Diabetes mellitus, $n(\%)$ & $71(28.5)$ & $20(24.7)$ & $43(32.6)$ & $8(22.2)$ & 0.309 \\
\hline Past stroke, $n(\%)$ & $36(14.5)$ & $13(13.6)$ & $21(15.9)$ & $4(11.1)$ & 0.740 \\
\hline Chronic renal failure, $n(\%)$ & $32(12.9)$ & $8(9.9)$ & $19(14.4)$ & $5(13.9)$ & 0.620 \\
\hline Parkinson's disease, $n(\%)$ & $11(4.4)$ & $5(6.2)$ & $5(3.8)$ & $1(2.8)$ & 0.624 \\
\hline Chronic lung disease, $n(\%)$ & $30(12.0)$ & $10(12.3)$ & $18(13.6)$ & $2(5.6)$ & 0.416 \\
\hline Smoking, $n(\%)$ & $7(2.8)$ & $3(3.7)$ & $3(2.3)$ & $1(2.8)$ & 0.828 \\
\hline $\begin{array}{l}\text { Peripheral vascular disease, } \\
n(\%)\end{array}$ & $13(5.2)$ & $4(4.9)$ & $6(4.5)$ & $3(8.3)$ & 0.657 \\
\hline \multicolumn{6}{|l|}{ Treatment: } \\
\hline Ventilated, $n(\%)$ & $18(7.2)$ & $5(6.2)$ & $10(7.6)$ & $3(8.3)$ & 0.894 \\
\hline No anticoagulation, $n(\%)$ & $10(4.0)$ & $1(1.2)$ & $7(5.3)$ & $2(5.6)$ & 0.299 \\
\hline
\end{tabular}




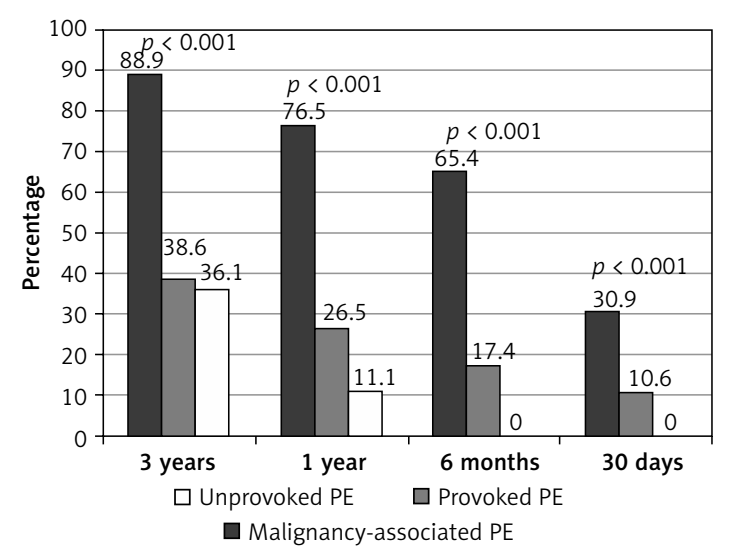

Figure 1 . Mortality rates in older adults with pulmonary embolism within 3 years of admission
Compared with survivors, patients who died within 1 year of admission had higher prevalence of dementia, congestive heart failure, diabetes mellitus, and active malignancy. They also had lower incidence of PPE and UPE (Table II). Regression analysis showed that UPE was independently associated with reduced 1-year mortality (Table III).

Relative to PPE and MAPE patients, the cumulative survival was significantly higher in UPE patients at each time point within 1 year of admission. However, 3 years after admission, the cumulative survival was comparable between PPE patients and UPE patients, and was significantly lower in MAPE patients (Figure 2).

Table II. Clinical characteristics of dead patients and survivors within the first year of admission

\begin{tabular}{|lcccc|}
\hline Parameter & $\begin{array}{c}\text { Survivals } \\
(n=148,59.4 \%)\end{array}$ & $\begin{array}{c}\text { Dead } \\
(n=101,40.6 \%)\end{array}$ & $\begin{array}{c}\text { Hazard ratio } \\
(95 \% \text { CI })\end{array}$ & $P$-value \\
\hline Age, mean \pm standard deviation [years] & $79.7 \pm 5.8$ & $80.0 \pm 5.6$ & NA & 0.718 \\
\hline Female, $n$ (\%) & $104(70.3)$ & $57(56.4)$ & $0.5(0.3-0.9)$ & 0.031 \\
\hline Hypertension, $n$ (\%) & $108(73.0)$ & $75(74.3)$ & $1.0(0.6-1.8)$ & 0.884 \\
\hline Ischemic heart disease, $n$ (\%) & $34(23.0)$ & $30(29.7)$ & $1.4(0.7-1.5)$ & 0.241 \\
\hline Atrial fibrillation, $n$ (\%) & $24(16.2)$ & $13(12.9)$ & $0.7(0.3-1.5)$ & 0.587 \\
\hline Dementia, $n$ (\%) & $13(8.8)$ & $18(17.8)$ & $2.2(1.0-4.8)$ & 0.049 \\
\hline Congestive heart failure, $n$ (\%) & $5(3.4)$ & $13(12.9)$ & $4.2(1.4-12.2)$ & 0.006 \\
\hline Diabetes mellitus, $n$ (\%) & $34(23.0)$ & $37(36.6)$ & $1.9(1.1-3.3)$ & 0.022 \\
\hline Past stroke, $n$ (\%) & $18(12.2)$ & $18(17.8)$ & $1.5(0.7-3.1)$ & 0.271 \\
\hline Chronic renal failure, $n$ (\%) & $18(12.2)$ & $14(13.9)$ & $1.1(0.5-2.4)$ & 0.704 \\
\hline Parkinson's disease, $n$ (\%) & $3(2.0)$ & $8(7.9)$ & $4.1(1.0-16.0)$ & 0.054 \\
\hline Chronic lung disease, $n(\%)$ & $16(10.8)$ & $14(13.9)$ & $1.3(0.6-2.8)$ & 0.553 \\
\hline Smoking, $n$ (\%) & $3(2.0)$ & $4(4.0)$ & $1.9(0.4-9.1)$ & 0.446 \\
\hline Peripheral vascular disease, $n$ (\%) & $6(4.1)$ & $7(6.9)$ & $1.7(0.5-5.4)$ & 0.388 \\
\hline Malignancy-associated PE, $n$ (\%) & $19(12.8)$ & $62(61.4)$ & $10.7(5.7-20.1)$ & $<0.001$ \\
\hline Provoked PE, $n$ (\%) & $97(65.5)$ & $35(34.7)$ & $0.2(0.1-0.4)$ & $<0.001$ \\
\hline Unprovoked PE, $n$ (\%) & $32(21.6)$ & $4(4.0)$ & $0.1(0.0-0.4)$ & $<0.001$ \\
\hline
\end{tabular}

Table III. Stepwise logistic regression analysis showing which variables were independently associated with mortality 1 year following admission

\begin{tabular}{|lccc|}
\hline Parameter & Hazard ratio & 95\% confidence interval & $P$-value \\
\hline Age & 1.1 & $1.0-1.1$ & 0.030 \\
\hline Dementia & 3.5 & $1.3-9.1$ & 0.011 \\
\hline Congestive heart failure & 9.9 & $2.8-36.1$ & $<0.001$ \\
\hline Diabetes mellitus & 2.4 & $1.2-4.9$ & 0.017 \\
\hline Malignancy-associated PE & 17.2 & $7.9-37.1$ & $<0.001$ \\
\hline Unprovoked PE & 0.3 & $0.1-1.0$ & 0.056 \\
\hline
\end{tabular}




\section{Discussion}

The incidence of UPE in adults with PE and its associated prognosis have already been studied before [1, 5-8]. However, to the best of our knowledge, the incidence and the prognosis of UPE in older adults have never been studied until now. According to our findings, UPE is not rare (14.5\%) in older adults with PE, and it is associated with a favorable prognosis within 1 year of admission in this population compared with PPE and MAPE. Moreover, UPE prognosis in older adults has been comparable to that of PPE towards the third year following admission.

According to our findings, the incidence of UPE in older adults with PE is lower than the incidence of UPE in adults with PE $-14.5 \%$ in older adults with PE compared with up to $50 \%$ in adults with $P E[1,7]-$ but it is not rare in older adults. Of note, the incidence of UPE in older adults with PE may be even lower; our findings may be an overestimation of the true incidence of UPE in older adults with $P E$, since only a few of the UPE patients in our study have undergone a genetic thrombophilia workup, and since according to Siguret et al. [9], $14.4 \%$ of older adults with venous thromboembolism have at least one inherited thrombophilia. The low incidence of UPE in older adults with PE may be explained by the high prevalence of malignancies, immobility, surgery, and osteoporotic fractures in this population.

At each time point within 3 years of admission mortality rates were highest and cumulative survival was lowest in older adults with MAPE compared with older adults with PPE and UPE. This finding is comparable with the long-term prognosis of adults with PE [7]. But contrary to adults with PE [7], UPE in older adults is associated with a better prognosis than PPE in older adults within the first year of admission. Moreover, these findings may be an underestimation, since patients with UPE in the current cohort were older than patients with PPE. In our opinion, UPE in older adults is probably provoked by minor triggers, such as mild dehydration or short immobility, which may have a small effect on the general prognosis. On the other hand, we assume that UPE in adults is probably provoked by more major triggers, such as an undiagnosed inherited thrombophilia, which might have a more substantial effect on the general prognosis [2]. Another explanation may be the devastating impact of the PPE trigger on the general prognosis of older adults compared with adults; for example, hip fracture might be associated with PPE in both older adults and adults, but in older adults it might also be associated with decubitus ulcers, delirium, urinary retention, and other complications which might affect prognosis, and are less frequent in adults [10].

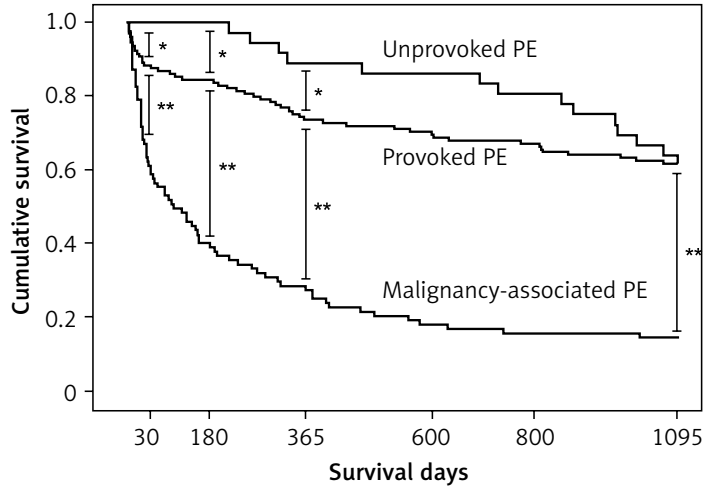

Figure 2. Cumulative survival in older adults with pulmonary embolism within 3 years of admission ${ }^{*} p<0.05,{ }^{* *} p<0.001$.

According to our findings, compared with PPE, UPE in older adults is associated with a better prognosis in terms of a higher cumulative survival within the first year of admission, but cumulative survival of UPE patients decreases and becomes equal to the cumulative survival of PPE patients during the next 2 years. We assume that the elevated mortality rates in PPE patients within the first year of admission are attributed to the PE, while the comparable mortality rates later in UPE patients and in PPE patients are attributed to co-morbidities other than the PE. Since data concerning anticoagulation therapy duration following discharge are missing in the current analysis, it is impossible to estimate the impact of the anticoagulation therapy duration on the long-term prognosis.

These findings may have significant clinical consequences: since UPE in older adults with PE is less common than in adults, but still not rare, and since the prognosis of PPE and MAPE is much worse in older adults than in other adults, physicians should look for a cause and especially for malignancy in older adults with PE. Paradoxically, many physicians pursue the diagnosis of malignancy only in younger PE patients, while actually they should pursue the diagnosis of malignancy in older adults with PE as well, since almost onethird of them have active malignancies according to the current analysis.

Except for the small sample size and the observational nature of the study, the main limitations of the current analysis are: a lack of information concerning the anticoagulation therapy duration following discharge; a lack of an inherited thrombophilia workup in most UPE patients; a lack of an occult malignancy workup in all UPE patients; a lack of information concerning how massive the PE was in terms of right heart failure; and a lack of information concerning the actual cause of death and PE-related deaths rather than an all-cause mortality. A lack of inherited thrombophilia and 
occult malignancy workup in older adults with UPE represents "real-life" medicine; physicians often do not perform these workups in older adults since the prevalence of hereditary thrombophilia declines in older age [11], and since there are no guidelines to look for an occult malignancy in older adults with UPE. Still, these limitations should be addressed in future large interventional prospective studies.

In conclusion, UPE incidence in older adults with PE is less common than in adults with PE, but it is not rare. UPE in older adults is associated with a better prognosis in terms of a higher cumulative survival - especially within the first year of admission.

\section{Conflict of interest}

The authors declare no conflict of interest.

\section{References}

1. Agnelli G, Becattini C. Acute pulmonary embolism. N Engl J Med 2010; 363: 266-74.

2. Heit JA, Spencer FA, White RH. The epidemiology of venous thromboembolism. J Thromb Thrombolysis 2016; 41: 3-14.

3. Spencer FA, Gore JM, Lessard D, et al. Venous thromboembolism in the elderly. A community-based perspective. Thromb Haemost 2008; 100: 780-8.

4. Spencer FA, Gurwitz JH, Schulman S, et al. Venous thromboembolism in older adults: a community-based study. Am J Med 2014; 127: 530-7.

5. Goldhaber SZ, Bounameaux H. Pulmonary embolism and deep vein thrombosis. Lancet 2012; 379: 1835-46.

6. White RH, Zhou H, Murin S. Death due to recurrent thromboembolism among younger healthier individuals hospitalized for idiopathic pulmonary embolism. Thromb Haemost 2008; 99: 683-90.

7. Lehmann R, Luxembourg B, Miesbach W, et al. Idiopathic (unexplained) pulmonary embolism is associated with an impaired prognosis compared to other entities of pulmonary embolism. Blood Coagul Fibrinolysis 2010; 21: 70-6.

8. Charlson ME, Cushman M. Epidemiology and risk factors for venous thrombosis. Semin Hematol 2007; 44: 62-9.

9. Siguret V, Emmerich J, Belleville T, et al. Prevalence of hereditary thrombophilia in patients older than 75 years with venous thromboembolism referred for thrombophilia screening. J Gerontol A Biol Sci Med Sci 2015; 70: 977-81.

10. Carpintero P, Caeiro JR, Carpintero R, Morales A, Silva S, Mesa M. Complications of hip fractures: a review. World J Orthop 2014; 5: 402-11.

11. Weingarz L, Schwonberg J, Schindewolf M, et al. Prevalence of thrombophilia according to age at the first manifestation of venous thromboembolism: results from the MAISTHRO registry. Br J Haematol 2013; 163: 655-65. 